\title{
The Sierpiński Sieve of Nim-varieties and Binomial Coefficients
}

\author{
Aviezri Fraenkel* \\ fraenkel@wisdom.weizmann.ac.il \\ Alex Kontorovich ${ }^{\dagger}$ \\ alexk@math.columbia.edu
}

July 27,2006

Dedicated to Ron Graham on his 70th birthday

\begin{abstract}
We consider the variety $X Y$, where instead of multiplication, we take the Nim-product. Its geometry turns out to be the Sierpiński sieve, which is well known to be connected to Pascal's triangle modulo two. We generalize Nim-sums and -products to what we call $q$-sums and -products for integers $q \geq 2$ (the original case corresponding to $q=2$ ). The Sierpiński sieve also generalizes to so-called $q$-sieves, and the original relationship extends completely. That is, the geometry of the $q$-variety $X Y$ is a $q$-sieve. The connection to binomial coefficients, though, only extends in the case where $q$ is prime, and we prove this using theorems of Kummer and Legendre.
\end{abstract}

\section{Introduction}

Mathematicians are quite accustomed to the fact that everything is connected to everything else. This paper is just another such instance. We analyze connections between fractals, binomial coefficients, and combinatorial games.

For the first topic, we deal with the famous Sierpiński triangle, or gasket, or sieve, shown in Fig. 1. Its construction is discussed in section 2.2.

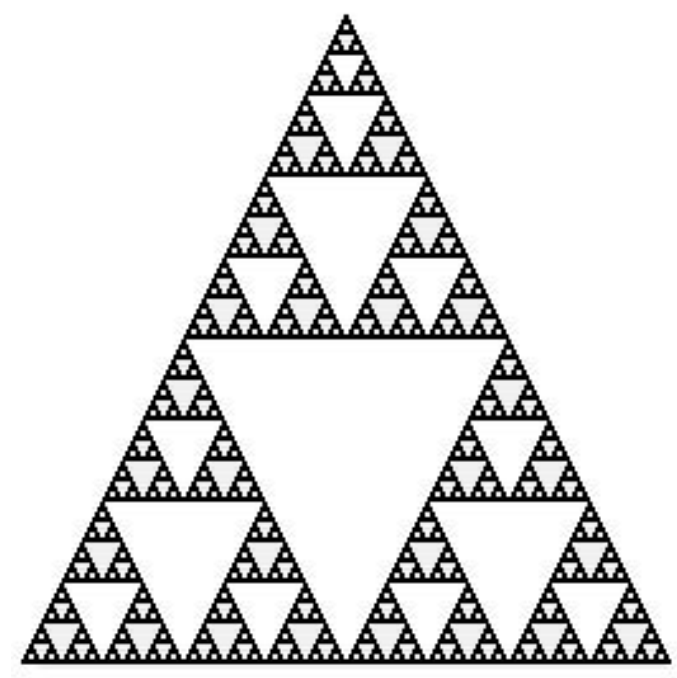

Figure 1: Sierpiński Triangle.

*Department of Computer Science and Applied Mathematics, Weizmann Institute of Science, Rehovot , Israel 76100

${ }^{\dagger}$ Department of Mathematics, Columbia University, New York, NY 10027 
For the second topic, we consider the equally famous triangle of Pascal ${ }^{1}$ (first ten rows shown in Fig. 2), known to be connected to cellular automata, Fermat's Last Theorem, and the prime recognition problem. It is also well known that the elements of Pascal's triangle modulo 2 form a Sierpiński sieve (see Fig. 3).

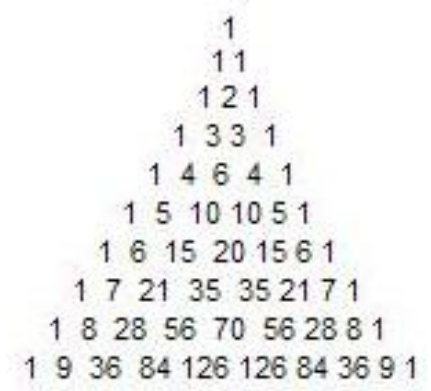

Figure 2: First ten rows of Pascal's Triangle.

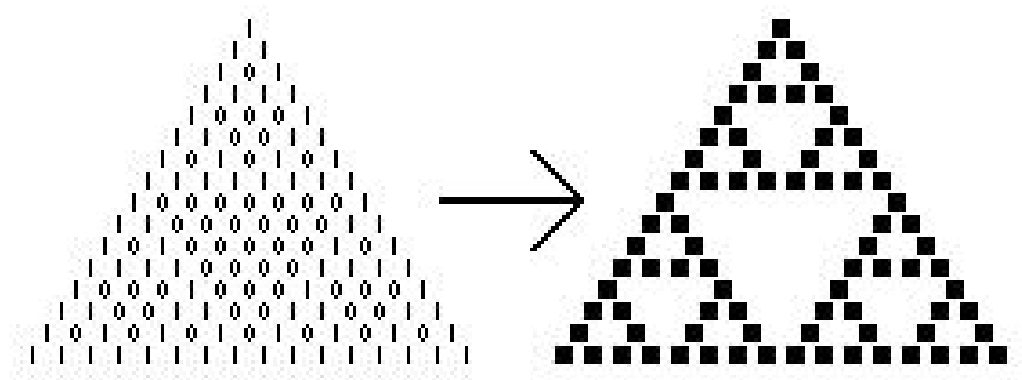

Figure 3: Pascal's triangle mod 2, with 0s represented by white dots and 1s by black dots.

Finally for the third topic, we consider the prototypical combinatorial game, Nim. Sit down at a table with a friend and make some piles out of chips, say three piles with 4,5 , and 7 chips per pile, respectively. Play proceeds by alternating moves between the two friends. A move consists of selecting a single pile, and removing as many chips as one desires from this pile, even the whole pile, but at least one chip. Say you remove 6 chips. Then the piles have 4, 5, and 1 chips each, and it is your friend's turn. Now you're playing Nim! The first player unable to make a legal move (because all of the chips have been removed from the table) loses, so the player to take the last chip off the table wins the game.

The strategy for this game is efficiently computable, and it induces two operations on the integers, namely the Nim-sum and Nim-product ${ }^{2}$. To compute the Nim-sum of nonnegative integers $x$ and $y$, simply write them in base 2 and add without carrying ${ }^{3}$. In other words, if $x=x_{0}+2 x_{1}+4 x_{2}+\ldots+2^{d} x_{d}$ and $y=y_{0}+2 y_{1}+4 y_{2}+\ldots+2^{d} y_{d}$, (of course requiring $x_{i}, y_{i} \in\{0,1\}$ ) then their Nim-sum, denoted $x \oplus y$, is defined by

$$
x \oplus y=n_{0}+2 n_{1}+\ldots+2^{d} n_{d},
$$

where $n_{i} \equiv x_{i}+y_{i}(\bmod 2), n_{i} \in\{0,1\}$. The Nim-product, denoted $x \otimes y$, is defined similarly by

$$
x \otimes y=w_{0}+2 w_{1}+\ldots+2^{d} w_{d},
$$

where $w_{i}=\left\{\begin{array}{cc}1, & x_{i}+y_{i} \geq 2 \\ 0, & \text { otherwise }\end{array}\right.$. (Since $x_{i}, y_{i} \in\{0,1\}$, we could have written $x_{i}+y_{i}=2$ instead of $\geq$ in the definition of $w_{i}$, or better yet just $w_{i}=x_{i} y_{i}$, but our version will generalize better.) It is clear that the bits $w_{i}$

\footnotetext{
${ }^{1}$ which was actually known over 700 years earlier to the Chinese mathematician Yang Hui, and at least 100 years before Pascal in the West. See [6].

${ }^{2}$ There is actually a third operation, Nim-multiplication, which is different from Nim-product. Together with Nim-summation, Nim-multiplication gives an alternate ring structure on the integers. Since the operation is not relevant to this paper, we will not define it.

${ }^{3}$ This is also called addition over GF (2), or XOR addition (exclusive OR).
} 
capture what we call the indicator carry, i.e., the instances when a carry is "forgotten" in the Nim-addition of $x$ and $y$. The following formula, relating ordinary addition, Nim-sums, and Nim-products, is a simple exercise:

$$
x+y=x \oplus y+2(x \otimes y) .
$$

What does this have to do with Nim? Well, it turns out that every position (by which we mean current list of number of chips in each pile, such as $\{4,5,7\}$, or $\{4,5,1\}$ as in our earlier example) falls into one of two categories - $\mathcal{P}$-positions (for Previous player winning) and $\mathcal{N}$-positions (for Next player winning; this will all make sense shortly). Every possible legal move from a $\mathcal{P}$-position results in an $\mathcal{N}$-position, and from every $\mathcal{N}$-position there is always at least one move to a $\mathcal{P}$-position. It turns out that the $\mathcal{P}$-positions are those for which the total Nim-sum of the numbers of chips is zero. So since $4 \oplus 5 \oplus 7=6,\{4,5,7\}$ is not a $\mathcal{P}$-position, while $\{4,5,1\}$ is a $\mathcal{P}$-position because $4 \oplus 5 \oplus 1=0$. Notice that once all the chips are gone, the position $\{0,0,0\}$ is a $\mathcal{P}$-position, and the Previous player (the one who moved to this position) has won the game by taking the last chip off the table. Thus a $\mathcal{P}$-position is one in which the Previous player can win, and so one strives to move to $\mathcal{P}$-positions (which is always possible from $\mathcal{N}$-positions, but impossible from $\mathcal{P}$-positions). So when it is your turn, look at the position and Nim-sum the number of chips in each pile. If you get anything bigger than zero, you're at an $\mathcal{N}$-position, so move to a $\mathcal{P}$-position (this choice can also be computed quickly, but this is not relevant for us here). If you are at a $\mathcal{P}$-position, well, your options are all $\mathcal{N}$-positions, from which your friend has a winning strategy - the best for you seems to be to take a single chip, to maximize the chances that your friend will make a mistake.

We begin our investigation by considering what we will call the Nim-variety $X Y$, or the set of numbers $x$ and $y$ such that $x \otimes y=0 .{ }^{4}$ In light of the formula (1), an equivalent definition is the set of $x$ and $y$ with full Nim-sum, i.e. whose Nim-sum is the same as the ordinary sum, $x+y=x \oplus y$. In some sense, this variety is the antithesis of the $\mathcal{P}$-positions, since instead of the zero Nim-sum required for a $\mathcal{P}$-position, we're requiring full Nim-sum, or equivalently, zero Nim-product. The genesis of our work was revealed to us while studying Fig. 4, which is a truncated picture of the variety. If you are not completely convinced that this picture is congruent to Fig. 1 (and Fig. 3) after a change of coordinates from Cartesian to those parametrizing Pascal's triangle, try tilting your head (or the paper) counterclockwise (resp. clockwise) $135^{\circ}$.

We consider the following generalizations. Sierpiński's triangle (itself a two-dimensional analogue of the Cantor Set) has been generalized to the tetrix (Fig. 5), pyramid (Fig. 6), carpet (Fig. 7), and sponge (Fig. 8) (pictures taken from [2], [9], [10], [11]). We include stages of the construction to justify our choice of calling these objects sieves (we are literally sieving out unwanted geometrical shapes at each stage, just like each stage of the sieve of Eratosthenes removes (unwanted) composites from the list of integers, leaving only the primes). The other reason for calling our generalizations sieves instead of, say, gaskets, is that gasket generalizations (Fig. 9 and 10) already appear in the literature (see [4], [12]) and are not the right generalizations for our purposes. In fact, these gaskets are precisely the nonzero points of Pascal's triangle modulo an arbitrary integer (compare Fig. 9 and 11). When this integer happens to be prime, the symmetry is perfect, but otherwise the figure is the superposition of the figures derived from the prime divisors (and even more complicated things happen when the integer is not squarefree). So on the primes, gaskets and sieves look the same, but the right generalization for us on composites will be sieves, not gaskets. Our so-called $q$-sieves will depend on an arbitrary integer $q \geq 2$ and are described in detail in Section 2. In Section 3, we extend Nim-sums and -products to what we call $q$-sums and $q$-products, and prove that the geometry of the $q$-variety $X Y$ (where multiplication is now a $q$-product) is a $q$-sieve. ${ }^{5}$ In Section 4, we consider instead of the nonzero terms of Pascal's triangle modulo two (already seen to be a Sierpiński sieve), the nonzero terms of Pascal's triangle modulo $q$. We recall and reprove (only for the sake of self-containment) the theorems of Legendre and Kummer, valid if and only if $q$ is a prime, and show that in this case, Pascal's triangle modulo $q$ is also a $q$-sieve. The figure below summarizes the connections discussed:

$$
\begin{aligned}
& q \text {-sieve iff } \\
& \| \quad q \text { prime } \\
& q \text {-variety } X Y================\text { Pascal's Triangle mod } q
\end{aligned}
$$

We conclude in Section 5 with open problems and directions of further research.

\footnotetext{
${ }^{4}$ Yes, we are playfully abusing terminology by calling this set a "variety". Another possibility is to call it the set of "zero-divisors". Besides, we are genuinely interested in the geometric properties of this set, so maybe variety is not so bad after all.

${ }^{5}$ For $q=2, q$-sum, $q$-product will be referred to as either Nim-sum, Nim-product, or 2-sum, 2-product in the sequel.
} 


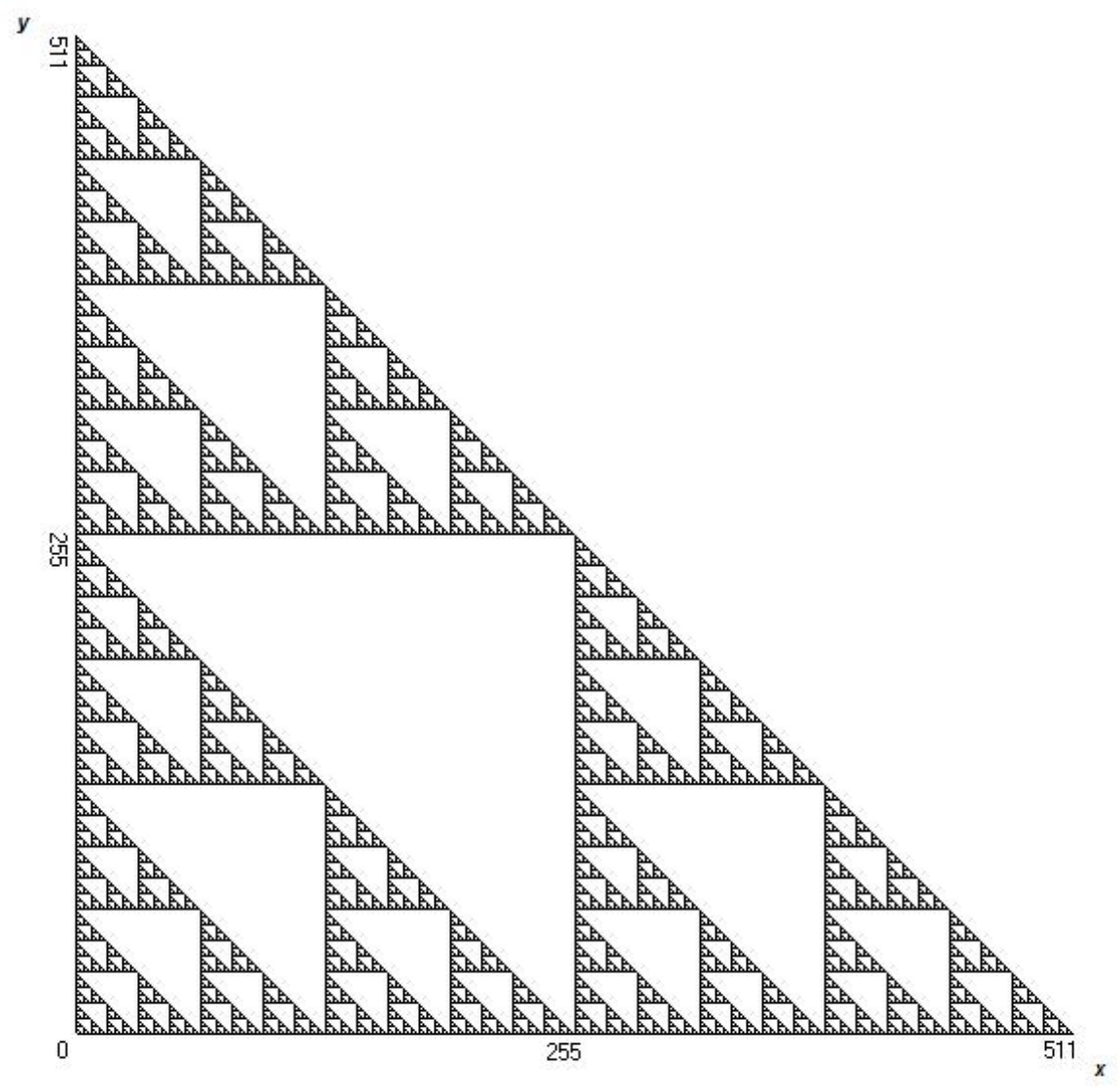

Figure 4: Black pixels mark elements of the set $\{(x, y): 0 \leq x, y<512, x \otimes y=0\}$.
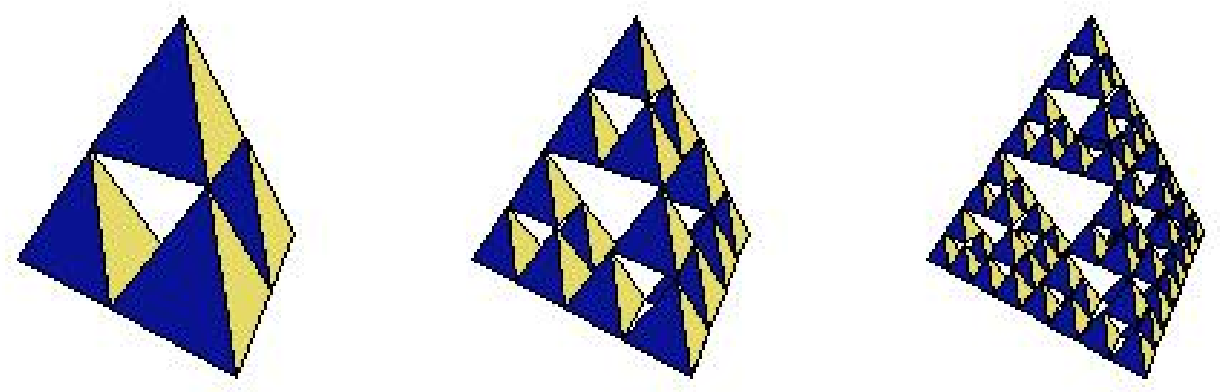

Figure 5: Sierpiński tetrix (from MathWorld [9]).

\section{Generalized Sierpiński Sieves}

\subsection{Cantor Set}

As a starting point, we recall the (sieve-like) construction of the Cantor Set. Consider the unit interval, $C_{0}=[0,1]$. Trisect it, removing the open middle third, and let $C_{1}$ denote the union of the remaining two closed intervals, $C_{1}=\left[0, \frac{1}{3}\right] \cup\left[\frac{2}{3}, 1\right]$. Repeat this operation (trisecting and sieving out the middle) on each of the remaining 

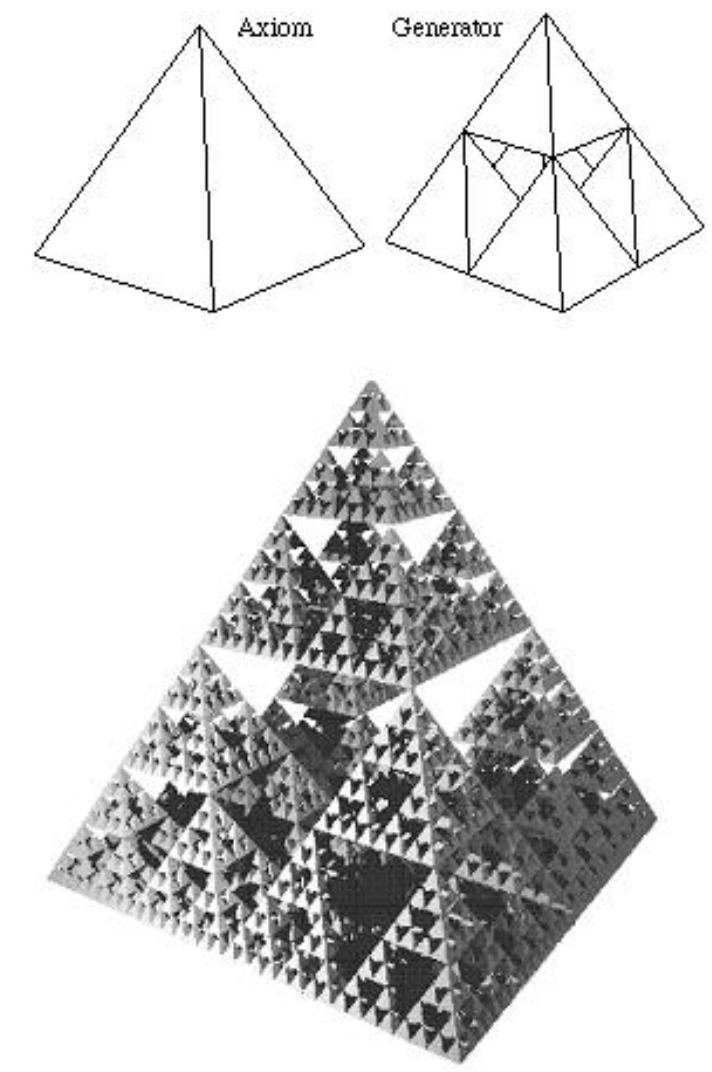

Figure 6: Sierpiński pyramid (from Bourke [2]).
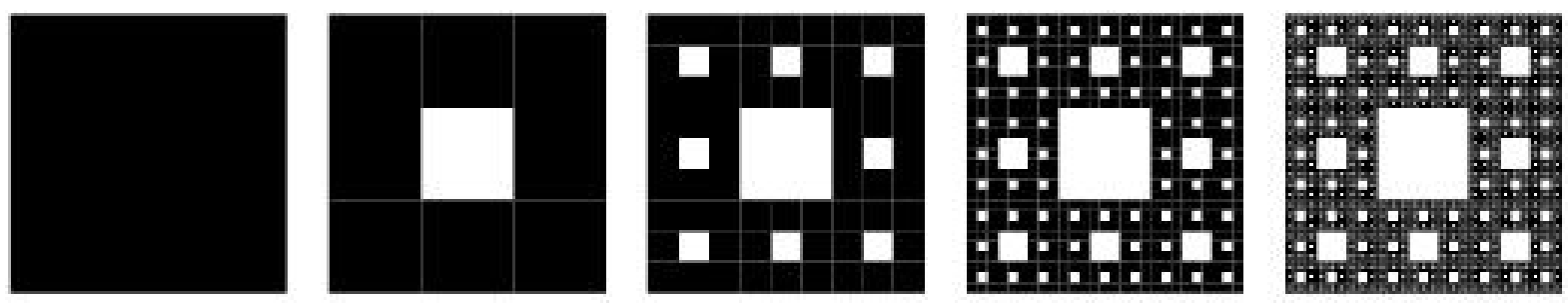

Figure 7: Sierpiński carpet (from MathWorld [11]).
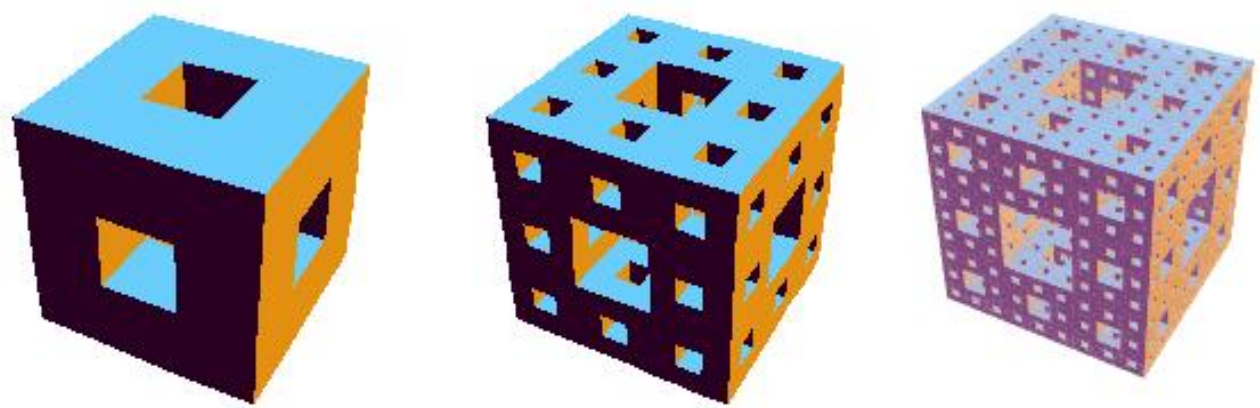

Figure 8: Sierpiński-Menger sponge (from MathWorld [10]). 


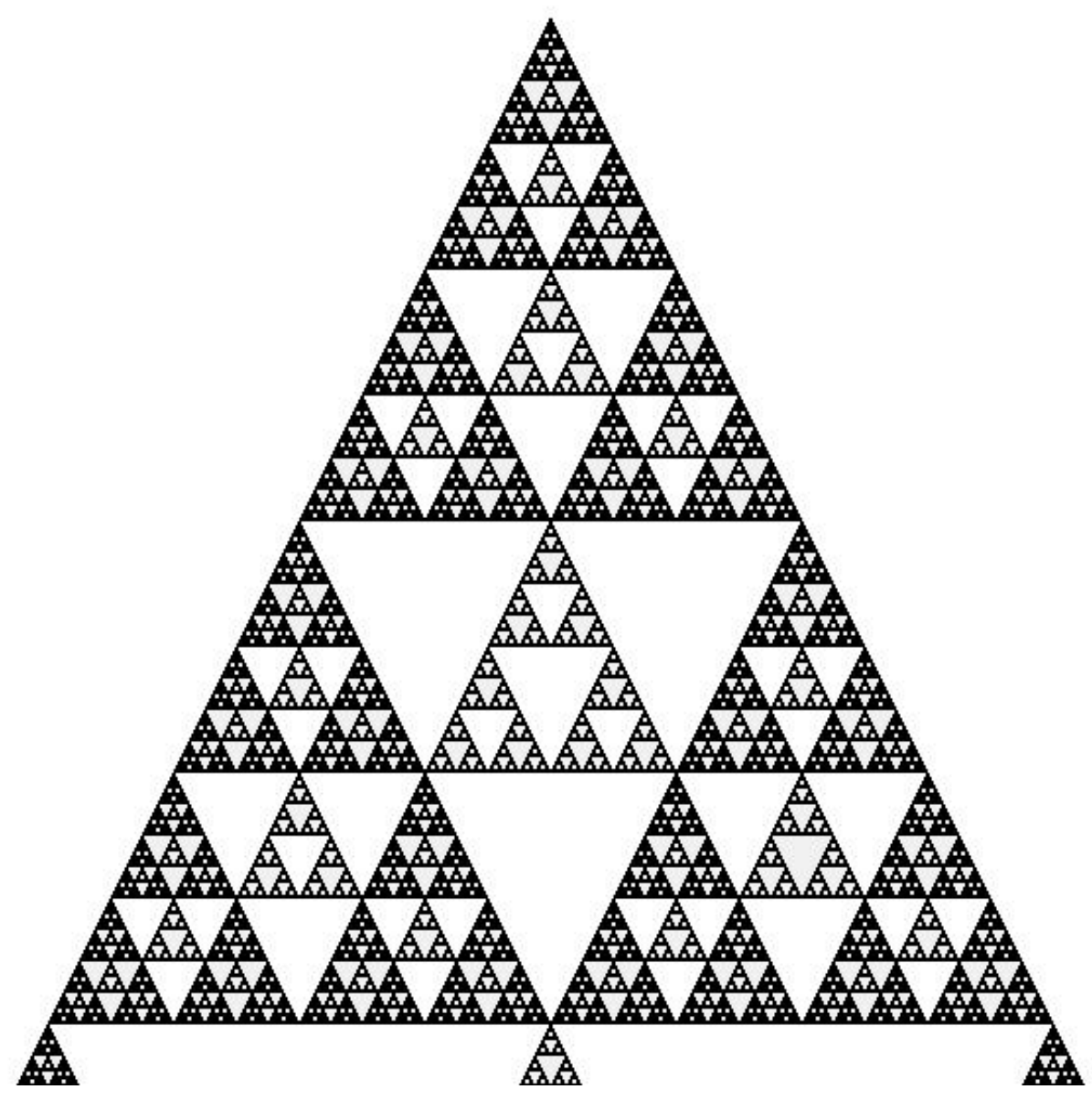

Figure 9: Generalized Sierpiński Gaskets: the 4-gasket.

intervals, leaving $C_{2}=\left[0, \frac{1}{9}\right] \cup\left[\frac{2}{9}, \frac{1}{3}\right] \cup\left[\frac{2}{3}, \frac{7}{9}\right] \cup\left[\frac{8}{9}, 1\right]$. Continue in this way, getting a decreasing sequence of closed sets, $C_{n}$, each the union of $2^{n}$ intervals, each interval of length $3^{-n}$. The remaining set $C=\bigcap_{n=1}^{\infty} C_{n}$ is the Cantor Set.

\subsection{Sierpiński Sieve}

\subsubsection{Geometric Construction}

We now construct the geometric version of the Sierpiński sieve as a two dimensional analog of the Cantor Set. See Fig. 12 to make the following verbal description clear.

We will call a triangle sitting if its base is horizontal,and standing if its top is horizontal (see Fig. 13 for the distinction). Consider the closed interior of a sitting equilateral triangle of unit side length. Denote this region by $S_{0}$. Connect bisectors of its sides, leaving three sitting triangles and one middle standing triangle. Remove the open standing triangle and denote the remaining region by $S_{1}$. Repeat this operation (bisecting sides and removing standing triangles) on each of the three remaining sitting triangles, leaving $S_{2}$, and so on. Continue in this fashion, getting a decreasing sequence of closed regions, $S_{n}$, each the union of $3^{n}$ equilateral sitting triangles, each triangle of side length $2^{-n}$. The set $S=\bigcap_{n=1}^{\infty} S_{n}$ is our old friend, the Sierpiński sieve. 


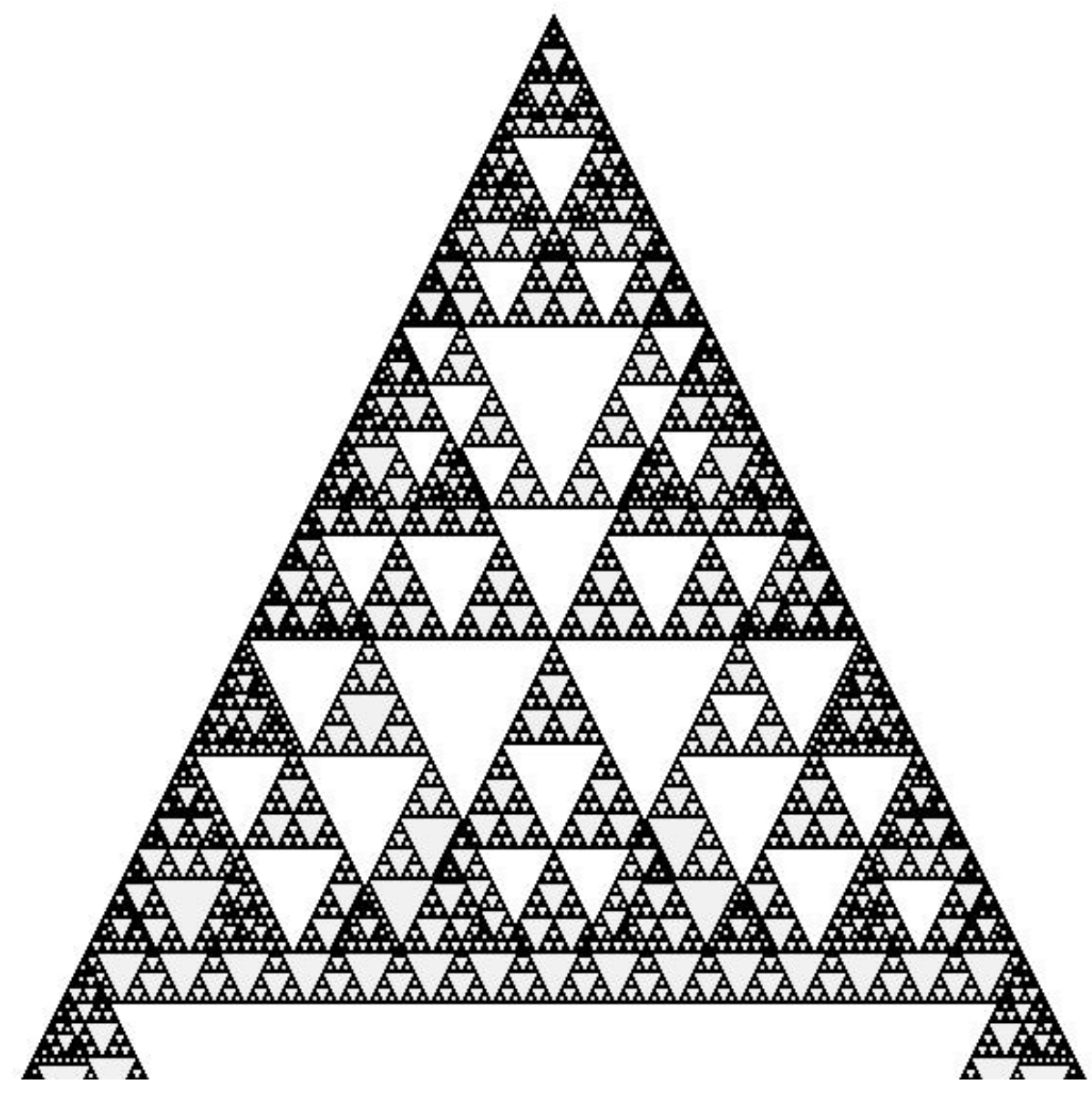

Figure 10: Generalized Sierpiński Gaskets: the 6-gasket.

\subsubsection{Analytic Construction}

To relate the picture of a sieve to the elements of a set, it will be necessary to have an analytic understanding of the same object, which we explain now. (This should be thought of as a discrete version of the geometric sieve.) Suppose $S \subset \mathbb{Z}_{\geq 0} \times \mathbb{Z}_{\geq 0}$ is any subset and denote by $S^{N}$ the truncation:

$$
S^{N}=\left\{(x, y) \in S: x+y<2^{N}\right\} .
$$

For any nonnegative integers $u$ and $v$, we denote by $S_{(u, v)}^{N}$ the truncated shifts:

$$
S_{(u, v)}^{N}=\left\{\left(x+u \cdot 2^{N}, y+v \cdot 2^{N}\right):(x, y) \in S^{N}\right\} .
$$

Notice that $S_{(0,0)}^{N}=S^{N}$, and that $S^{0}$ either consists of just the origin or is empty, depending on whether $S$ contains the origin. With this notation, we can define the analytic notion of the sieve:

Definition 1 A set $S$ is called the Sierpinski sieve iff

1. $S$ contains the origin, $(0,0) \in S$.

2. For all $N \geq 0$, the following recurrence decomposition holds:

$$
S^{N+1}=\bigcup_{u+v<2} S_{(u, v)}^{N}=S^{N} \cup S_{(1,0)}^{N} \cup S_{(0,1)}^{N} .
$$

The triangles in the union above are precisely those called sitting in the geometric definition, and the equality above (as opposed to just inclusion) corresponds to the removal of the standing triangles. 


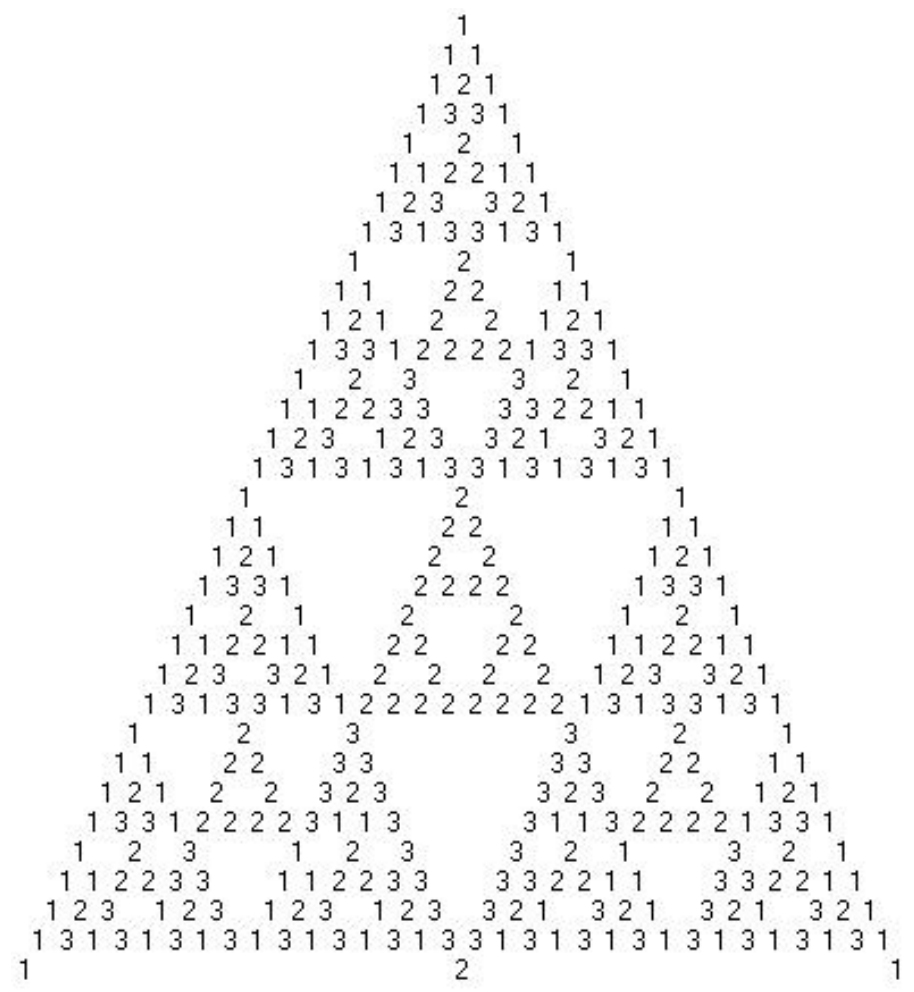

Figure 11: Pascal's Triangle mod 4 .
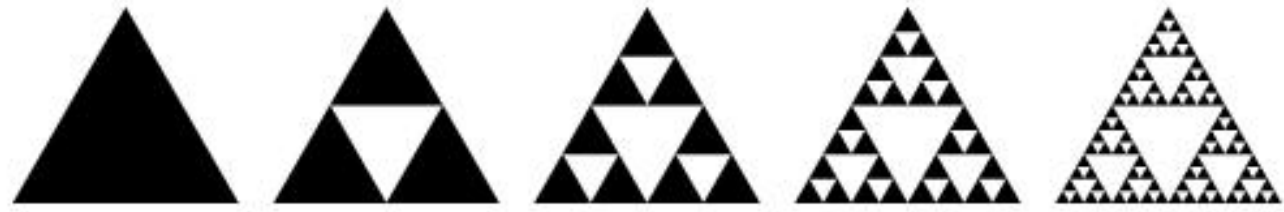

Figure 12: The Sierpiński Sieve (from MathWorld [8]).

a)

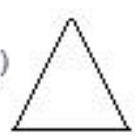

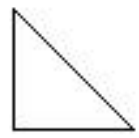

b)
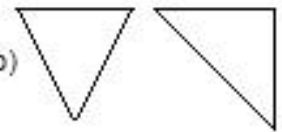

Figure 13: a) sitting triangles, b) standing triangles.

\section{$2.3 \quad q$-Gaskets}

Though we will not relate any of our work here to $q$-gaskets, it is important to distinguish them from $q$-sieves. Rather than delve into a lengthy discussion, we will content ourselves with defining $q$-gaskets to be the set of non-zero elements of Pascal's triangle $\bmod q$ (plotting these points analogously to the plot of Pascal's triangle mod 2). We point the reader to [3] from where Figures 9 and 10 were generated.

\section{$2.4 \quad q$-Sieves}




\subsubsection{Geometric Construction}

We now fix an integer $q \geq 2$, and construct a generalization of the Sierpiński sieve. Begin once again with the closed interior of a sitting equilateral triangle of unit side length, denoted by $G_{0}$. Divide each side into $q$ pieces of equal size and connect corresponding points. This creates $\frac{q(q+1)}{2}$ sitting triangles and $\frac{q(q-1)}{2}$ standing triangles. We remove all of the standing triangles and call the remaining region $G_{1}$. Repeat this operation on each of the $\frac{q(q+1)}{2}$ remaining sitting triangles, leaving $G_{2}$, and so on. Continuing in this way, we get a decreasing sequence of closed regions, $G_{n}$, each the union of $\left(\frac{q(q+1)}{2}\right)^{n}$ equilateral sitting triangles, each triangle of side length $q^{-n}$. Call the set $G=\bigcap_{n=1}^{\infty} G_{n}$ the $q$-sieve. Of course, the Sierpiński sieve is nothing but the 2 -sieve. Notice the perfect symmetry, even for composite $q$, the key difference between our sieve and gaskets (see Fig. 14).
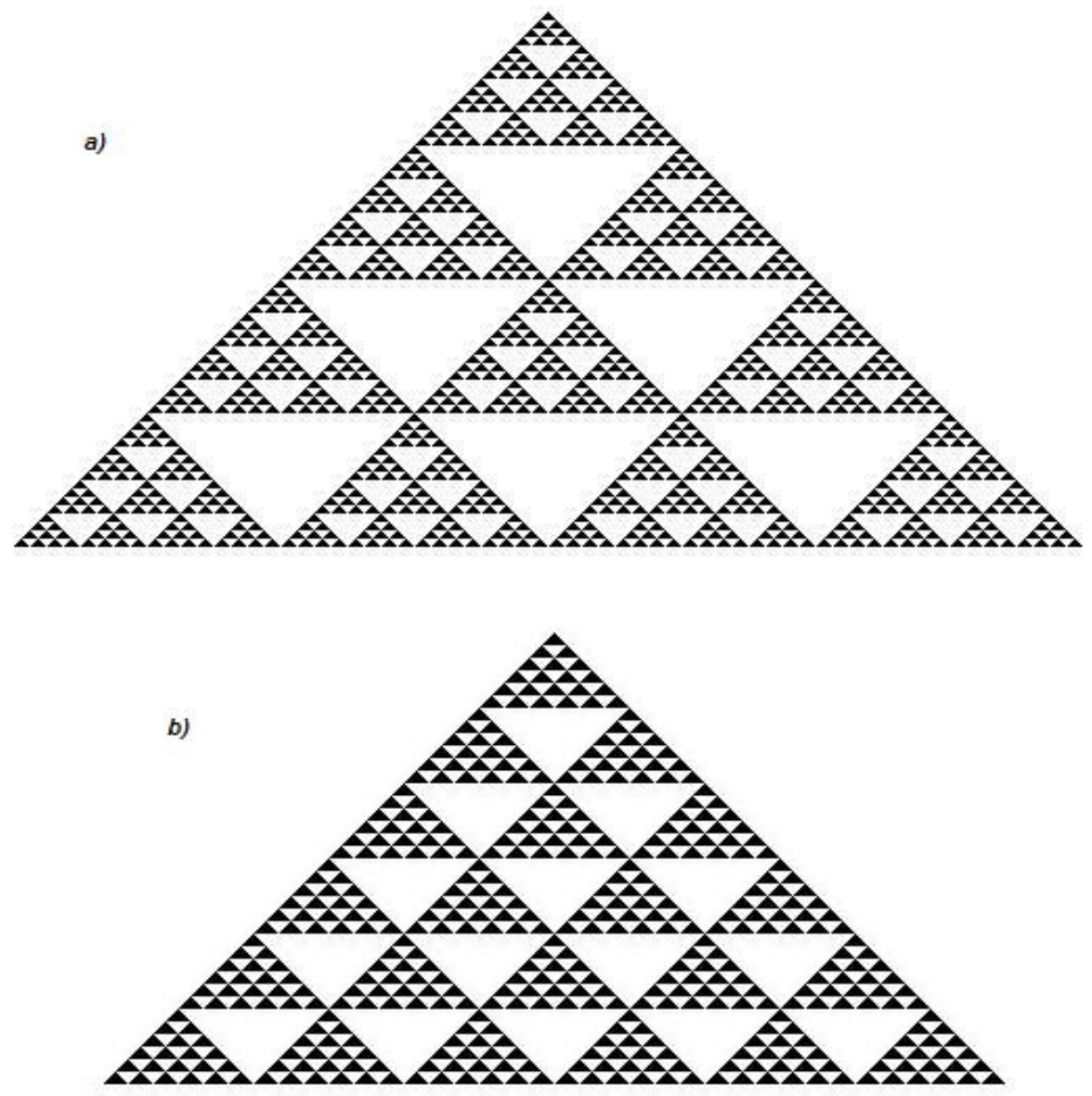

Figure 14: a) 4-sieve b) 6-sieve.

\subsubsection{Analytic Construction}

As before, we give the discrete version of the $q$-sieve. For a subset $S \subset \mathbb{Z}_{\geq 0} \times \mathbb{Z}_{\geq 0}$, we denote by $S^{N}$ the truncation:

$$
S^{N}=\left\{(x, y) \in S: x+y<q^{N}\right\}
$$

and the shifts:

$$
S_{(u, v)}^{N}=\left\{\left(x+u \cdot q^{N}, y+v \cdot q^{N}\right):(x, y) \in S^{N}\right\}
$$


for nonnegative integers $u$ and $v$.

Definition $2 S$ is the q-sieve iff it contains the origin and if for all $N \geq 0$, we have:

$$
S^{N+1}=\bigcup_{u+v<q} S_{(u, v)}^{N}
$$

Here the union is over $\frac{q(q+1)}{2}$ shifted truncations corresponding to the sitting triangles (the number of nonnegative $u$ and $v$ with sum no more than $q$ ). See Fig. 15 .

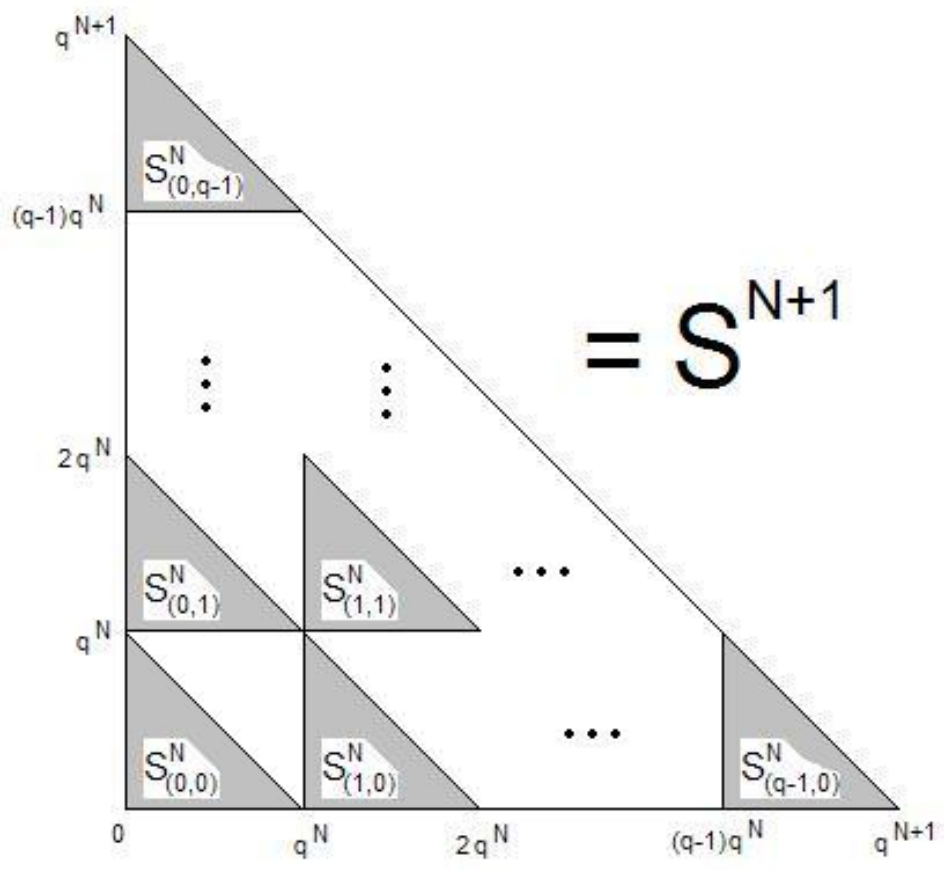

Figure 15: The $q$-sieve construction.

\section{$3 \quad q$-Sums and $q$-Products}

\subsection{Definitions}

Recall from Section 1 the definitions of the Nim-sum and Nim-product of non-negative integers $x$ and $y$, denoted $x \oplus y$ and $x \otimes y$, respectively. Write $x$ and $y$ in base 2 , so $x=x_{0}+2 x_{1}+4 x_{2}+\ldots+2^{d} x_{d}$, and $y=y_{0}+2 y_{1}+$ $4 y_{2}+\ldots+2^{d} y_{d}$, with $x_{i}, y_{i} \in\{0,1\}$. Then define

$$
x \oplus y=n_{0}+2 n_{1}+4 n_{2}+\ldots+2^{d} n_{d},
$$

where $n_{i} \equiv\left(x_{i}+y_{i}\right)(\bmod 2), n_{i} \in\{0,1\}$, and

$$
x \otimes y=w_{0}+2 w_{1}+4 w_{2}+\ldots+2^{d} w_{d},
$$

with $w_{i}=\left\{\begin{array}{cc}1 & x_{i}+y_{i} \geq 2 \\ 0 & \text { otherwise }\end{array}\right.$. Also recall equation (1):

$$
x+y=x \oplus y+2(x \otimes y) .
$$

The variety that we are interested in is the zero-set of the polynomial $X Y$, or in other words, the set $\{(x, y): x \otimes y=0\}$, which we recall is the same as the set of full Nim-sum: $\{(x, y): x+y=x \oplus y\}$. 
Now, fix an integer $q \geq 2$. Denote the $q$-sum and $q$-product of nonnegative integers $x$ and $y$ by $x \oplus_{q} y$ and $x \otimes_{q} y$, respectively, and define them analogously as follows. Write $x$ and $y$ in base $q$, so $x=x_{0}+q x_{1}+q^{2} x_{2}+\ldots+q^{d} x_{d}$, and $y=y_{0}+q y_{1}+q^{2} y_{2}+\ldots+q^{d} y_{d}$, with $x_{i}, y_{i} \in\{0,1, \ldots, q-1\}$. Then define

$$
x \oplus_{q} y=n_{0}+q n_{1}+q^{2} n_{2}+\ldots+q^{d} n_{d},
$$

where $n_{i} \equiv\left(x_{i}+y_{i}\right)(\bmod q), n_{i} \in\{0,1, \ldots, q-1\}$, and

$$
x \otimes_{q} y=w_{0}+q w_{1}+q^{2} w_{2}+\ldots+q^{d} w_{d},
$$

with $w_{i}=\left\{\begin{array}{cc}1, & x_{i}+y_{i} \geq q \\ 0, & \text { otherwise }\end{array}\right.$. Again, the $w_{i}$ are the indicator carry in the $i$-th place of the $q$-sum of $x$ and $y$, (in particular, now we see why we wanted $\geq$ instead of $=$ in the original definition of $w_{i}$ ) and the same argument proves that:

$$
x+y=\left(x \oplus_{q} y\right)+q\left(x \otimes_{q} y\right) .
$$

Our new variety is really the same old variety, $X Y$, but now we call it the $q$-variety and unravel the formalism to see that we want to study the set of zero $q$-products, $\left\{(x, y): x \otimes_{q} y=0\right\}$. The answer to the analogous question of geometry of $q$-varieties should be clear from Fig. 16 and $17-q$-varieties are $q$-sieves. Notice that even for $q$ composite (such as $q=4$ and $q=6$ ) we get the perfect symmetry of sieves instead of the broken pictures of gaskets (compare with Fig. 9 and 10).

\subsection{All varieties are sieves}

Let $S \subset \mathbb{Z}_{\geq 0} \times \mathbb{Z}_{\geq 0}$ be the variety of interest:

$$
S=\left\{(x, y): x \otimes_{q} y=0\right\} .
$$

Theorem $3 S$ is a q-sieve.

Proof. By the definition in section 2.4.2, we only need to show that $(0,0) \in S$ (obvious) and the formula:

$$
S^{N+1}=\bigcup_{u+v<q} S_{(u, v)}^{N}
$$

Unraveling the formalism, we just need that if $x$ and $y$ satisfy both $x+y<q^{N}$ and $x \otimes_{q} y=0$, then the product $\left(x+u \cdot q^{N}\right) \otimes_{q}\left(y+v \cdot q^{N}\right)$ vanishes for nonnegative integers $u$ and $v$ satisfying $u+v<q$. Looking at the definition of $w_{N}$ after (5), this statement is obvious, and we have inclusion of the union in the larger truncation. The opposite inclusion follows just as easily, and we are done.

\section{Modular Binomial Coefficients}

Pascal's triangle is made up of the binomial coefficients, where the $k$ th entry in the $n$th row is $\left(\begin{array}{c}n \\ k\end{array}\right)=$ $\frac{n !}{k !(n-k) !}$, with $m !=1 \cdot 2 \cdot \ldots \cdot(m-1) \cdot m$ (just ordinary multiplication, not Nim-products). By the Fundamental Theorem of Arithmetic, any integer $n$ is uniquely decomposable into a product of prime powers, i.e., $n=$ $p_{1}^{e_{1}} p_{2}^{e_{2}} \ldots p_{f}^{e_{f}}$. Let $e_{p}(n)$ denote the exponent with which $p$ appears in the prime decomposition of $n$, i.e. if $e_{p}(n)=k$, then $p^{k} \mid n$ but $p^{k+1} \nmid n$. Recall Legendre's 1808 result regarding the exact power of the prime $p$ dividing $n$ !: 


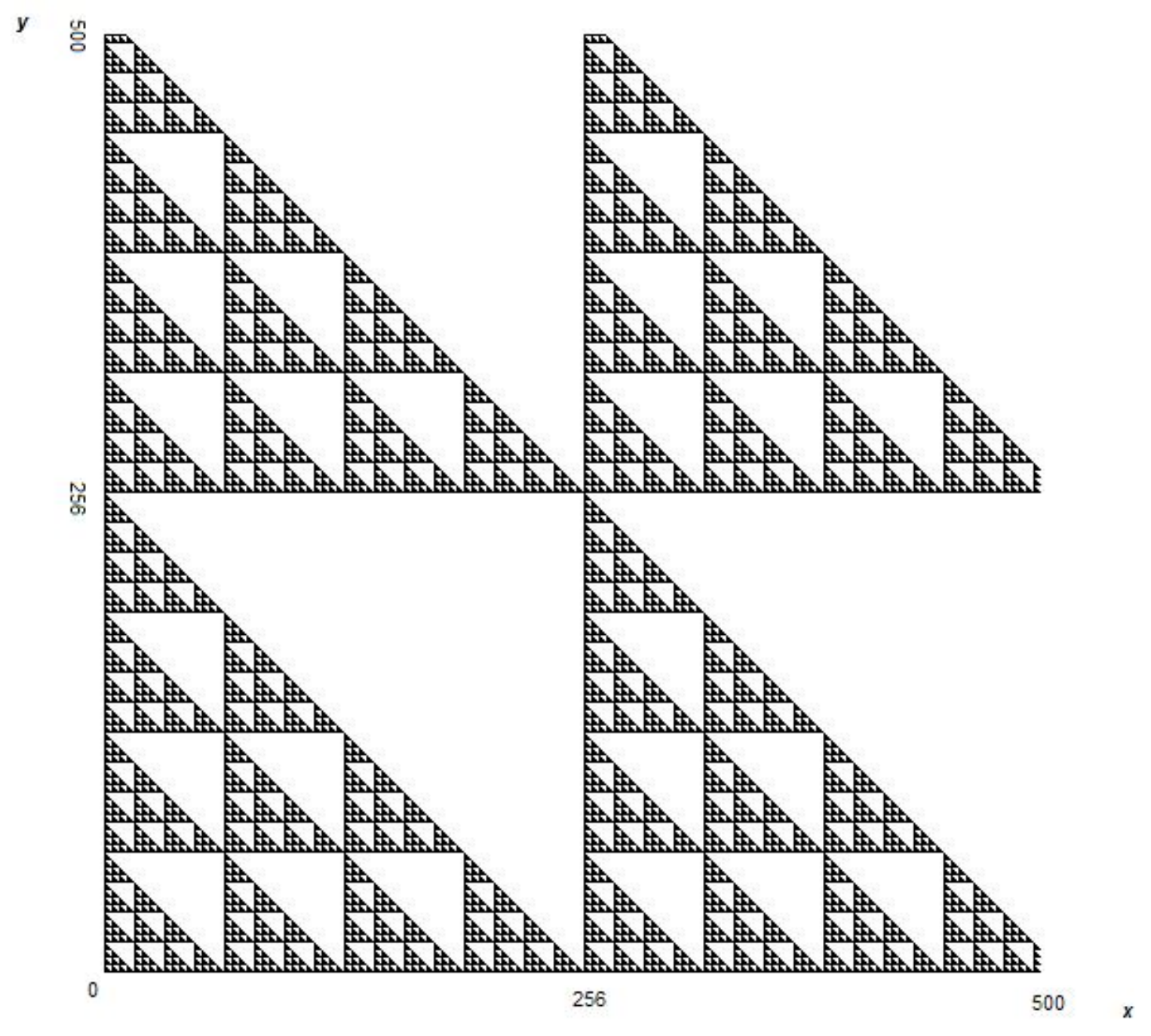

Figure 16: Black pixels mark elements of the 4-variety: $\left\{(x, y): 0 \leq x, y<500, x \otimes_{4} y=0\right\}$.

Theorem 4 (Legendre) $e_{p}(n !)=\left\lfloor\frac{n}{p}\right\rfloor+\left\lfloor\frac{n}{p^{2}}\right\rfloor+\left\lfloor\frac{n}{p^{3}}\right\rfloor+\ldots$, where $\lfloor z\rfloor$ is the floor function, returning the greatest integer less than or equal to $z$.

Proof. The first term on the right hand side counts the number of $m \leq n$ which are divisible by $p$. The second term takes into account that some of these $m$ are also divisible by $p^{2}$, and so have an extra contribution to $e_{p}(n !)$, and so on.

And now for Kummer's 1852 result:

Theorem 5 (Kummer) Let $p$ be prime and suppose $x+y=n$. Then $e_{p}\left(\left(\begin{array}{l}n \\ x\end{array}\right)\right)$, the exact power of $p$ dividing the binomial coefficient $\left(\begin{array}{c}n \\ x\end{array}\right)$, is the number of carries in the ordinary base $p$ addition of $x$ and $y$.

Proof. Recall that we write $n$ in base $p$ as $n=n_{0}+p n_{1}+p^{2} n_{2}+\ldots+p^{d} n_{d}$, and similarly with $x$ and $y$, and let $z_{i}=\left\{\begin{array}{cc}1, & x_{i}+y_{i}+z_{i-1} \geq q \\ 0, & \text { otherwise }\end{array}\right.$ (with the convention $z_{-1}=0$ ), measuring whether there occurs what 


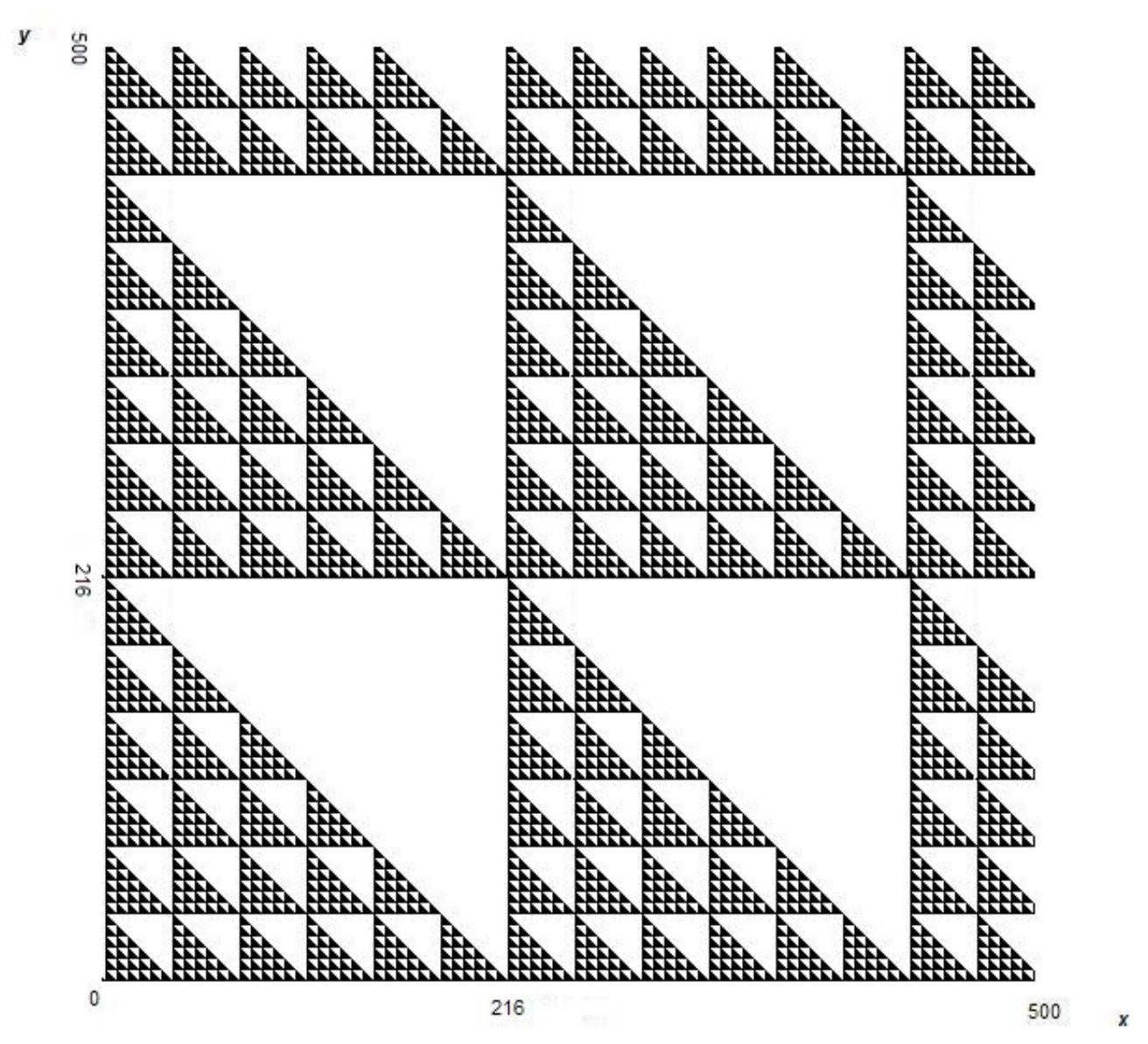

Figure 17: Black pixels mark elements of the 6-variety: $\left\{(x, y): 0 \leq x, y<500, x \otimes_{6} y=0\right\}$.

we call a propagating carry in the $i$ th place of the ordinary sum of $x$ and $y$. ${ }^{6}$ We will need the sum-of-digits function, defined by $\sigma_{p}(n)=n_{0}+n_{1}+\ldots+n_{d}$. Our first claim is that $(p-1) e_{p}(n !)=n-\sigma_{p}(n)$. Notice that $\left\lfloor\frac{n}{p}\right\rfloor=n_{1}+p n_{2}+p^{2} n_{3}+\ldots+p^{d-1} n_{d}$, while $\left\lfloor\frac{n}{p^{2}}\right\rfloor=n_{2}+p n_{3}+\ldots+p^{d-2} n_{d}$, etc. Putting this together with Legendre's theorem, it is straightforward to verify:

$$
\begin{aligned}
(p-1) e_{p}(n !) & =(p-1) n_{1}+\left(p^{2}-1\right) n_{2}+\left(p^{3}-1\right) n_{3}+\ldots+\left(p^{d}-1\right) n_{d} \\
& =n-\sigma_{p}(n) .
\end{aligned}
$$

A few more moment's thought leaves us with:

$$
(p-1) e_{p}\left(\left(\begin{array}{c}
n \\
x
\end{array}\right)\right)=\sigma_{p}(x)+\sigma_{p}(y)-\sigma_{p}(n),
$$

since $x+y=n$. But clearly $n_{0}=x_{0}+y_{0}-p z_{0}$ and $n_{i}=x_{i}+y_{i}-p z_{i}+z_{i-1}$ for $i \geq 1$, so:

$$
\begin{aligned}
e_{p}\left(\left(\begin{array}{l}
n \\
x
\end{array}\right)\right) & =\frac{1}{p-1}\left(x_{0}+y_{0}-\left(x_{0}+y_{0}-p z_{0}\right)+\sum_{i=1}^{d}\left(x_{i}+y_{i}-\left(x_{i}+y_{i}-p z_{i}+z_{i-1}\right)\right)\right) \\
& =\sum_{i=0}^{d} z_{i},
\end{aligned}
$$

${ }^{6}$ One should be careful not to confuse (as we did - we thank Don Knuth for bringing this to our attention) the propagating carry in ordinary addition, $z_{i}$ and the indicator carry in $q$-sums, $w_{i}$, as defined after (5). For instance in base 10 , the ordinary sum $54+46=100$ has two propagating carries, while the 10 -sum $54 \oplus_{10} 46=90$ has but one indicator carry. The $z_{i}$ and $w_{i}$ may only disagree if there are two consecutive carries. Fortunately, the situation we are studying requires no carries, so there is no problem. 
which is the total number of propagating carries.

Remark. Notice that the arguments above all required $p$ to be prime, due to the use of the Fundamental Theorem of Arithmetic. This is really the obstruction to equality between $q$-sieves and $q$-gaskets for composite $q$.

With Kummer's theorem in hand, it is obvious that $p$ does not divide the binomial coefficient $\left(\begin{array}{c}x+y \\ x\end{array}\right)$ (i.e. $\left(\begin{array}{c}x+y \\ x\end{array}\right)$ is nonzero modulo $p$ ) if and only if the number of (both indicator and propagating) carries is zero (meaning that the Nim-product is zero and we are on the variety). Notice finally that we are not looking at the coefficient $\left(\begin{array}{l}x \\ y\end{array}\right)$, but instead $\left(\begin{array}{c}x+y \\ x\end{array}\right)=\left(\begin{array}{c}x+y \\ y\end{array}\right)$. This is explained by the necessity of the $135^{\circ}$ head turn between figures of varieties (like 3,16, and 17) and sieves (like 2, 14a, and 14b) or equivalently a change of coordinates from Cartesian to those parametrizing Pascal's triangle. See Fig 18 for Pascal's triangle modulo the prime 3. This concludes our findings.
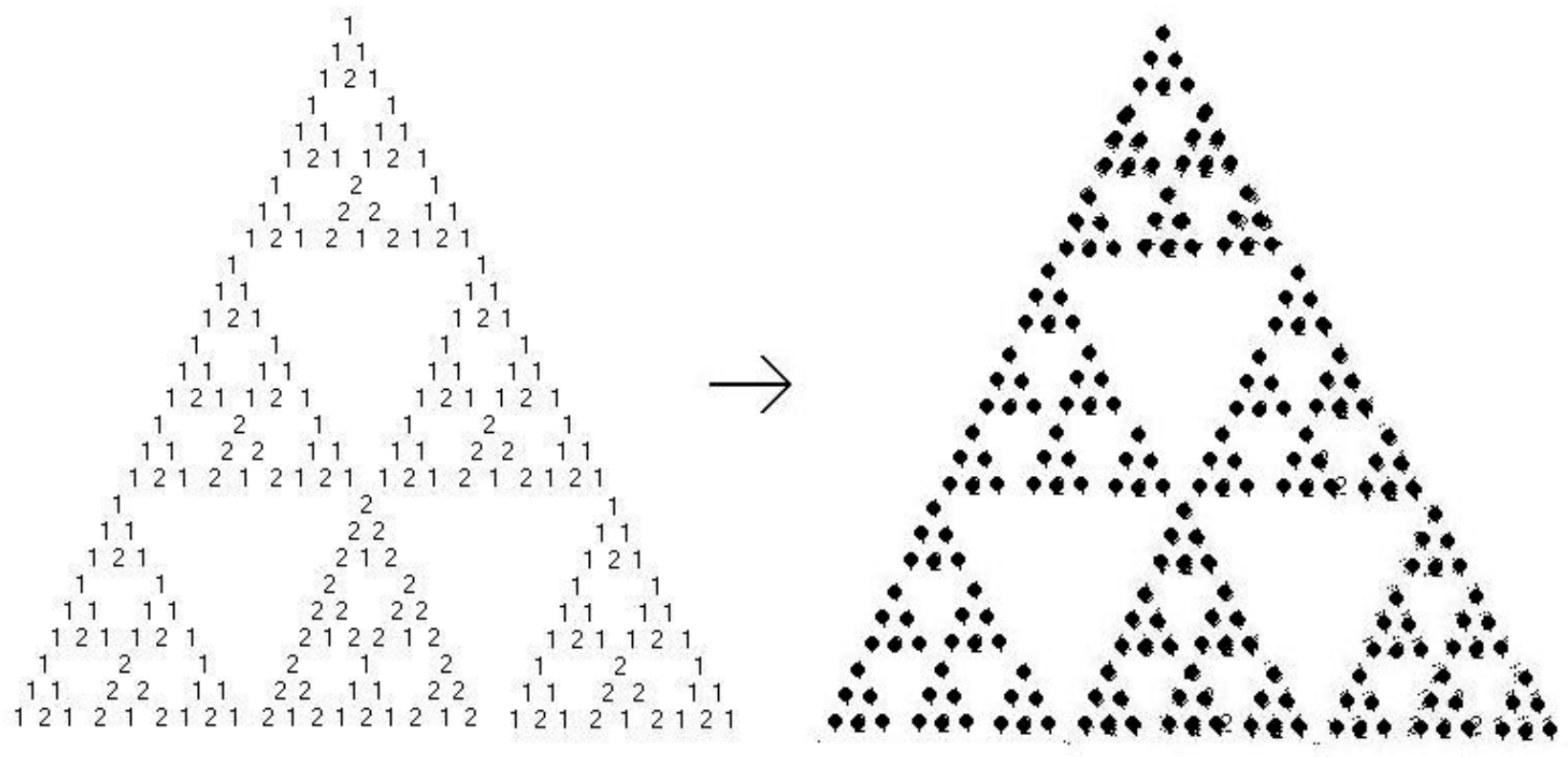

Figure 18: Pascal's triangle mod 3 .

\section{Conclusion}

Let us return to the diagram from the Introduction:

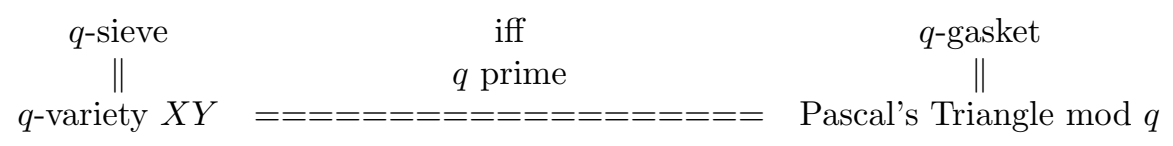

We have seen that the $q$-variety is geometrically the $q$-sieve, and through Kummer's theorem, so is Pascal's Triangle $\bmod q$ (but only when $q$ is prime). Here are some further questions for research:

- There are several analogs to Pascal's Triangle in higher dimensions, which we suspect have the geometry of the Sierpiński tetrix or pyramid. 
- The 2-sum gives $\mathcal{P}$-positions for an actual combinatorial game, namely Nim. Moore's $\operatorname{Nim}_{q}[7],[1]$, Ch. 15, is the same as Nim, except that a move consists of taking chips from up to $q$ piles where $q \geq 1$ is a fixed parameter. $\mathrm{Nim}_{1}$ is regular Nim. To win in $\mathrm{Nim}_{q}$, express every pile in binary, but sum them modulo $q+1$. The $\mathcal{P}$-positions are precisely those for which this sum is 0 . A take-away game whose winning strategy depends on expressing the pile sizes in a base $q>2$ and summing $\bmod q$ was proposed in [5].

- (Posed by Noam Zeilberger.) What is the geometry of the level sets of $X Y$, i.e. the variety generated by $x \otimes y-m=0$ for some positive integer $m$ ? We believe these pictures to be superpositions of $q$-sieves, much in the same way that a composite $q$-gasket is a superposition of the gaskets of its divisors.

Acknowledgment. The authors express their gratitude to Paul Bourke and MathWorld for permission to use their images, and to Don Knuth for his kind attention to detail. The first author thanks Tom Ferguson for providing reference [5]. (James A. Flanigan, a practicing physician, got his Ph.D. in math under Ferguson, published 6 papers, and then went on to get an M.D. degree.) The second author thanks the Weizmann Institute for support during this work, and Leonid Kontorovich and Noam Zeilberger for useful comments.

\section{References}

[1] E. R. Berlekamp, J. H. Conway and R. K. Guy, Winning Ways for your Mathematical Plays, 2 vols., Academic Press, London, 1982.

[2] P. Bourke. "Sierpiński Gasket." http://astronomy.swin.edu.au/ pbourke/fractals/gasket/

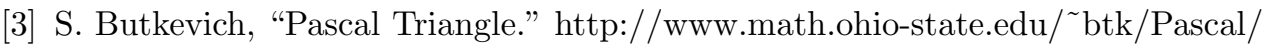

[4] A. Granville, Arithmetic properties of binomial coefficients. I. Binomial coefficients modulo prime powers, Organic mathematics (Burnaby, BC, 1995), 253-276, CMS Conf. Proc., 20, Amer. Math. Soc., Providence, RI, 1997.

[5] J. A. Flanigan, NIM, TRIM and RIM, unpublished manuscript, available from http://www.math.ucla.edu/ tom/papers/unpublished.html

[6] M. Hazewinkel (editor), Encyclopaedia of Mathematics, Vol. 7, "Pascal Triangle", Kluwer, 1991.

[7] E. H. Moore, A generalization of the game called nim, Ann. of Math. (Ser. 2) 11 (1909-1910) 93-94.

[8] E. W. Weisstein. "Sierpiński Sieve." From MathWorld-A Wolfram Web Resource. http://mathworld.wolfram.com/SierpinskiSieve.html

[9] E. W. Weisstein. "Tetrix." From MathWorld-A Wolfram Web Resource. http://mathworld.wolfram.com/Tetrix.html

[10] E. W. Weisstein. "Menger Sponge." From MathWorld-A Wolfram Web Resource. http://mathworld.wolfram.com/MengerSponge.html

[11] E. W. Weisstein. "Sierpiński Carpet." From MathWorld-A Wolfram Web Resource. http://mathworld.wolfram.com/SierpinskiCarpet.html

[12] S. Wolfram, Geometry of Binomial Coefficients, Amer. Math. Monthly, 91 (1984), 566-571. 